\title{
IMPACTO DE LA SALUD BUCAL SOBRE LA CALIDAD DE VIDA EN ESCOLARES DE LA ESCUELA "SAN FRANCISCO DE PELEUSÍ DE AZOGUES". 2016
}

\section{IMPACT OF ORAL HEALTH ON THE QUALITY OF LIFE IN SCHOOL CHILDREN OF THE SCHOOL "SAN FRANCISCO DE PELEUSÍ DE AZOGUES". 2016}

\author{
León-Matovelle Erika ${ }^{1}$, Delgado-López María ${ }^{2}$, Aguilar-Maldonado José ${ }^{3}$, Crespo-Crespo Cristina ${ }^{4}$ \\ ${ }^{1}$ Odontóloga Universidad Católica de Cuenca sede Azogues. Ecuador \\ 2 Odontóloga Especialista Docente Universidad Católica de Cuenca Sede Azogues. Ecuador \\ ${ }^{3}$ Odontólogo Especialista Docente Universidad Católica de Cuenca Sede Azogues. Ecuador \\ ${ }^{4}$ Dra. Mg. Docente Universidad Católica de Cuenca Sede Azogues. Ecuador
}

\begin{abstract}
Resumen
OBJETIVO: El objetivo de esta investigación fue conocer el impacto de la salud bucal sobre la calidad de vida mediante el CPQ (Cuestionario de Percepción de Salud Bucal) corto. MATERIALES Y METODOS: El estudio fue realizado en niños de 6 a 10 años de la Escuela General Básica "San Francisco de Peleusí de Azogues"mediante el "Cuestionario de Percepción de Salud Bucal" o "CPQ corto" que está formado por 4 dimensiones ("Sintomatología Oral, Limitación Funcional, Bienestar Emocional y Bienestar Social”). A cada escolar se le explicó la pregunta y marcaron la respuesta de acuerdo a su afectación. RESULTADOS: Los problemas que afectaron con mayor frecuencia fueron: comida atrapada entre sus dientes $(54,5 \%)$, sangrado en las encías $(22,7 \%)$, de esta manera la dimensión que presentó mayor prevalencia fue: La de Sintomatología Oral, en la cual se encontró significancia estadística $(\mathrm{p}=0,03)$, seguida de limitación funcional $(\mathrm{p}=0,021)$ y bienestar social $(\mathrm{p}=0,013)$. CONCLUSIÓN: Las afecciones de la salud oral influyeron negativamente en la calidad de vida de los escolares en lo que respecta al problema comida atrapada entre los dientes y la dimensión más afectada fue la de Sintomatología Oral, en cuanto a sexo el femenino presento más problemas de salud bucal afectando su calidad de vida.
\end{abstract}

Palabras clave: salud bucal, calidad de vida, CPQ corto, bienestar social.

\begin{abstract}
The aim of this investigation was to determine the impact of oral health in the quality of life using the short CPQ. MATERIALS AND METHODS: The study was done in children of 6 to 10 years of the Escuela General Básica "San Francisco de Peleusí de Azogues", was used the Perceptions Questionnaire Oral Health or short CPQ, the same that consists of 4 dimensions (Oral Symptoms, Limitation functional, Emotional Welfare and Social Welfare).Previously each question was explained at the students and after the answer was marked according their involvement.RESULTS: The most frequent problems were: food trapped between the teeth (54.5\%), bleeding in the gums (22.7\%), in this way the Dimension that presented the highest prevalence was Oral Symptomatology, in $(P=0.03)$, followed by functional limitation $(p=0.021)$ and social welfare $(p=$ 0.013). CONCLUSION: The oral health problems affect negatively the quality of life of the students, Respect to the food problems trapped between the teeth and the most affected dimension was the Oral Symptomatology; It is female who has more oral health problems.
\end{abstract}

Key words: oral health, quality of life, short CPQ, oral symptoms, emotional welfare, social welfare.

\section{INTRODUCCIÓN}

La calidad de vida se define como el estado de bienestar personal que nos permite asociarnos completamente en las funciones y actividades que nos relacionan con aspectos físicos, sociales y psicológicos adecuados para cada edad. ${ }^{1}$ De esta manera consideramos que la salud oral, es un factor fundamental de la salud integral, siendo parte importante del nivel de calidad de vida. ${ }^{2}$ Moreno y cols en el año 2014 definen a calidad de vida en relación con la salud bucal, 
(CVRSB) abarcando 4 condiciones que son psicológicas, sociales, funcionales y la presencia de dolor o molestias. ${ }^{3}$ Actualmente el concepto de salud necesita tener inmersos aspectos psicosociales, como cuestiones relacionadas a la calidad de vida e influyendo en las relaciones humanas: ${ }^{4}$ así como las percepciones de una persona en el contexto social. ${ }^{5}$ La salud bucodental es un factor concluyente para la calidad de vida ${ }^{5}$ y las enfermedades orales conllevan una afectación de la misma a niños en edad preescolar, interfiriendo en su crecimiento, el peso, la socialización, la autoestima, el aprendizaje, y también en la calidad de vida de sus padres. ${ }^{6}$ El dolor es un síntoma que también afecta la calidad de vida. Por lo que tiene interés conocer la etiología del dolor bucofacial que puede ser causado por distintas patologías como la caries dental, los traumatismos dentales y las maloclusiones. Entonces se puede decir que la etiología de este dolor es multifactorial y, aunque la maloclusión por si, no causa dolor buco-facial, puede dar lugar a dolor de forma indirecta al provocar la patología de la articulación temporo-mandibular y traumatismos en los tejidos bucales duros y blandos. ${ }^{7}$ En este sentido podríamos señalar que la calidad de vida bucodental es la autopercepción que tiene la persona en relación a su salud bucal, al comer, relacionarse, e igualmente su estética. ${ }^{8}$ Al-Omiri y cols en el 2010 indicaron que las enfermedades bucales afectaron varios aspectos del diario vivir. ${ }^{9}$ La salud bucodental tradicionalmente es percibida y tratada como una entidad separada del resto del organismo humano en la práctica clínica (Huang, 2013), sin embargo, está asociada no sólo en el aspecto fisiológico con el resto del organismo, sino que influye psicológicamente en la autopercepción que tienen los individuos acerca de su salud y capacidad de enfrentarse a las distintas situaciones diarias. (Kaufman y cols. 2014). ${ }^{10}$ En este sentido, se diseña la siguiente investigación realizada en niños de la Escuela General Básica "San Francisco de Peleusí de Azogues". El objetivo de esta investigación es conocer de qué manera se ve influenciada la salud oral en relación con la vida cotidiana de los niños de modo que se pueda intervenir sobre sus efectos, para ello se han desarrollado a lo largo de los años varios índices con la intención de objetivar la calidad de vida como aspecto subjetivo; uno de ellos es el Cuestionario de Percepción de Salud Oral (The Child Perceptions Questionnaire) o CPQ. Fue desarrollado por Jokovic y colaboradores en el 2002, es un instrumento que mide la CVRSB en niños, en edades comprendidas entre 8 a 10 años y 11 a 14 años. El cuestionario se aplicó a niños entre 8 y 10 años; consta de 29 preguntas de las cuatro semanas anteriores, existen versiones cortas de 16 y 8 preguntas: ${ }^{11}$ las respuestas se registran en una escala Likert: $0=$ nunca, $1=$ una 0 dos veces, $2=$ a veces, $3=$ a menudo 4 $=$ todos los días o casi todos los días. ${ }^{12}$ La mayor puntuación en el CPQ8-10 es de 100 y el mínimo es de 0, esto indica que a mayor puntaje, peor Calidad de Vida. El CPQ es un cuestionario de auto-reporte desarrollado para medir la salud bucodental relacionada con la calidad de vida. El cuestionario tiene como objetivo mejorar la descripción de la salud oral de los niños, teniendo en cuenta la importancia de los aspectos psicológicos en el concepto de salud. ${ }^{13}$

\section{MATERIALES Y MÉTODOS}

Se realizó un estudio de tipo descriptivo transversal con 163 niños de 6 a 10 años matriculados en la Escuela de Educación General Básica "San Francisco de Peleusí de Azogues", en el período 2016, de ambos sexos y que cumplieron con los parámetros de selección, especialmente lo concerniente a firmas de consentimiento informado y asentimiento informado. El estudio tuvo un nivel de confiabilidad del $95 \%$ y un error de $5 \%$ y un valor de $\mathrm{p}<0.05$. La muestra ajustada a pérdidas fue de 172 niños; sin embargo luego de aplicar los criterios de selección, la muestra final quedó constituida por 163 niños. El tipo de muestreo fue probabilístico aleatorio simple. Se inició con la entrega de los consentimientos informados a cada padre de familia , obteniendo los consentimientos informados y asentimientos por parte del niño; posterior a ello se procedió a realizar el cuestionario a los niños de segundo a sexto de básica, explicándoles que constan de 2 partes, la primera de datos generales del paciente edad y sexo, la segunda consta de datos del CPQ corto. Se indicó lo que quería decir cada pregunta para que marquen la respuesta correcta. Los datos obtenidos se analizaron en el programa Excel versión 2010 elaborando una base de datos con el respectivo diccionario; para el análisis estadístico se utilizó el paquete estadístico SPSS versión 19, obteniendo tablas de frecuencias absolutas y relativas, y el análisis bivariado se realizó mediante la prueba de Chi cuadrado.

La información se resumió por medio de tablas y gráficos que permitieron mostrar los resultados de los 163 niños comprendidos en edades de 6 a 10 años, utilizando los parámetros de dicho cuestionario, para observar la relación entre salud bucal y calidad de vida.

\section{Resultados}

Se encuestaron a 163 escolares. En cuanto a la Distribución de frecuencia según necesidad de salud bucal percibida, en niños de la Escuela de Educación Básica "San Francisco de Peleusí de Azogues", muestra que en la opción de respuesta SIEMPRE la pregunta más prevalente fue en la Dimensión de Sintomatología Oral con la \# 4: Tiene problemas de comida atorada entre los dientes con un $36,20 \%$, seguida de la pregunta \# 2. Sangrado en sus encías al comer o cepillarse los dientes con un $22,70 \%$ y por último, la Dimensión de Limitación Funcional con la pregunta \# 5. Problemas para comer cosas duras con un $14,11 \%$. (tabla 1) Según la Identificación del impacto de la Salud oral sobre la Calidad de Vida de acuerdo al "CPQ corto", según la edad en el rango de 67 años en la Escuela de Educación Básica "San Francisco de Peleusí de Azogues",: en la opción de respuesta SIEMPRE, la 
Dimensión más prevalente fue en la de Sintomatología Oral con la pregunta \# 4: "Tiene problemas de comida atorada entre los dientes" con un 54,5\% , seguida de la pregunta \# 2: Sangrado en sus encías al comer o cepillarse los dientes con un $22,7 \%$, y, por último, la Dimensión de Limitación Funcional con la pregunta \# 5: Problemas para comer cosas duras con un 20,5\%, compartiendo el mismo valor en la Dimensión de Bienestar Emocional con la pregunta \# 11: "Le preocupa lo que sus compañeros piensen de sus dientes" con un 20,5\%. Se encontró significancia estadística $(\mathrm{p}=0,03)$ en la pregunta \# 4: Comida atorada entre dientes que corresponde a la dimensión de Sintomatología Oral. (Tabla 2). Al Evaluar "Impacto de la Salud Bucal sobre Calidad de Vida" de acuerdo al "CPQ corto", según edad en el rango de 8-10 años en la Escuela de Educación Básica "San Francisco de Peleusí de Azogues", en la opción de respuesta SIEMPRE la Dimensión más prevalente fue: en la de Sintomatología Oral con la pregunta \# 4: Tiene problemas de comida atorada entre los dientes con un $29,4 \%$, seguida de la pregunta \# 2 : Sangrado en sus encías al comer o cepillarse los dientes con un 22,7 \%, y por último, la Dimensión de Limitación Funcional con la pregunta \# 5: Problemas para comer cosas duras compartiendo, el mismo valor con la pregunta \# 6: "Dolor de muela al comer alimentos muy fríos o muy calientes" con un $11,8 \%$. Se encontró significancia estadística $(\mathrm{p}=0,03)$ en la pregunta \# 4: Comida atorada entre dientes que corresponde a la dimensión de Sintomatología Oral. (Tabla 3). En el Análisis del impacto de la Salud Bucal sobre la Calidad de Vida de acuerdo al "CPQ corto"según el sexo masculino en la Escuela de Educación Básica "San Francisco de Peleusí de Azogues". En la opción de respuesta SIEMPRE, la Dimensión más prevalente fue en la de Sintomatología Oral con la pregunta \# 4: Tiene problemas de comida atorada entre los dientes con un $27,8 \%$, seguida de la pregunta \# 2: Sangrado en sus encías al comer o cepillarse los dientes con un $22,2 \%$ y por último la Dimensión de Limitación Funcional con la pregunta \# 5: Problemas para comer cosas duras con un 15,3\%. Se encontró significancia estadística $(\mathrm{p}=0,021)$ en la pregunta \# 7: Problemas para pronunciar algunas palabras que corresponde a la dimensión de Limitación Funcional, seguida de la pregunta \# 16. Dientes son motivo de pelea con una significancia estadística de $(\mathrm{p}=0,013)$ que corresponde a la dimensión de Bienestar Social. (Tabla 4) En la Identificación del "impacto de la Salud Bucal sobre la Calidad de Vida" de acuerdo al "CPQ corto" según el sexo femenino en la Escuela de Educación Básica "San Francisco de Peleusí de Azogues". En la opción de respuesta SIEMPRE la Dimensión más prevalente fue en la de Sintomatología Oral con la pregunta \# 4: Tiene problemas de comida atorada entre los dientes con un 42,9\%, seguida de la pregunta \# 2: Sangrado en sus encías al comer o cepillarse los dientes con un $23,1 \%$ y por último la Dimensión de Bienestar Emocional con la pregunta \# 11. Le preocupa lo que sus compañeros piensen de sus dientes con un $18,6 \%$. Se encontró significancia estadística $(\mathrm{p}=0,021)$ en la pregunta \# 7. Problemas para pronunciar algunas palabras que corresponde a la Dimensión de Limitación Funcional, seguida de la pregunta \# 16. Dientes son motivo de pelea con una significancia estadística de $(\mathrm{p}=0,013)$ que corresponde a la dimensión de Bienestar Social. (Tabla 5)

\section{DISCUSIÓN}

El siguiente estudio se realizó con el objeto de conocer el impacto de la salud bucal sobre calidad de vida en escolares entre 6 y 10 años, mediante el "Cuestionario de Percepción de Salud Bucal" o "CPQ corto" con 4 dimensiones: ("Sintomatología Oral, Limitación Funcional, Bienestar Emocional, Bienestar Social"). ${ }^{12}$ En el estudio se puedo evidenciar que las Dimensiones más afectadas fueron Sintomatología Oral $(36,20 \%)$ y Limitación Funcional $(14,11 \%)$, presentando altos porcentajes al igual que los estudios de Barbosa y cols 14 y Moreno y cols ${ }^{3}$

En cuanto a los resultados obtenidos en la investigación la prevalencia de las afecciones fueron 3: en la dimensión de Sintomatología Oral en las preguntas \# 4. Comida atorada entre los dientes con un $54,5 \%$, y la pregunta \# 2. Sangrado de encías al comer o cepillarse los dientes con un 22,7\%, en la dimensión de Limitación Funcional en la pregunta \# 5. Tiene problemas para comer cosas duras con un $14,11 \%$, mientras que en el estudio de Barbosa y cols, ${ }^{14}$ las dimensiones de mayor prevalencia fueron Sintomatología Oral, Bienestar Emocional y Bienestar Social con valores similares a este estudio; por otro lado, Jocovic y col, ${ }^{15}$ afirman que las dimensiones de mayor incidencia fueron Sintomatología Oral y Bienestar Emocional con valores similares a los de este estudio.

En el presente estudio se encontró significancia estadística en la Dimensión de Sintomatología Oral con un $(\mathrm{p}=0,03)$, la dimensión de limitación funcional con un $(\mathrm{p}=0,021)$, y por último la dimensión de bienestar social con un $(\mathrm{p}=0,013)$, contrastando con Aguilar y col ${ }^{16}$ en el año 2011 quienes determinaron una correlación significativa en los dominios de: síntomas orales $(\mathrm{p}=0,049)$ y bienestar emocional ( $\mathrm{p}$ $=0,022$ ). Con los datos obtenidos en esta investigación se observa que la salud bucal tiene una gran relación con la calidad de vida, pero en un mayor porcentaje en las mujeres. se pudo observar adicionalmente un inconveniente en el rango de edad de 6-7 años, debido a que los niños no comprendieron adecuadamente las preguntas, por lo que se les explicó de tal forma que respondan cada pregunta con la respuesta correcta en relación a sus afecciones; sin embargo se puede asumir que pudo haber cierto grado de subjetividad, por lo que sería importante concluir que este instrumento es más ajustado para una población de 8 años en adelante en donde los resultados podrían ser más objetivos.

\section{CONCLUSIÓN}

El problema comida atrapada entre los dientes según el "CPQ corto", fue el más reportado por los escolares y 


\begin{tabular}{|c|c|c|c|c|c|c|c|c|c|c|}
\hline \multicolumn{11}{|c|}{$n=163$} \\
\hline \multirow{2}{*}{ PREGUNTAS } & \multicolumn{2}{|c|}{ A VECES } & \multicolumn{2}{|c|}{$\begin{array}{c}\text { CASI } \\
\text { NUNCA }\end{array}$} & \multicolumn{2}{|c|}{$\begin{array}{l}\text { CASI } \\
\text { SEMPRE }\end{array}$} & \multicolumn{2}{|c|}{ NUNCA } & \multicolumn{2}{|c|}{ SIEMPRE } \\
\hline & $\mathbf{n}$ & $\%$ & $\mathbf{n}$ & $\%$ & $\mathbf{n}$ & $\%$ & $\mathbf{N}$ & $\%$ & $\mathbf{n}$ & $\%$ \\
\hline \multicolumn{11}{|l|}{ Sintomatologia oral } \\
\hline 1 Dolor molar & 45 & 27,61 & 6 & 3,68 & 17 & 10,43 & 80 & 49,08 & 15 & 9,20 \\
\hline $\begin{array}{l}2 \text { Sangrado en sus encias al comer o cepillarse los } \\
\text { dientes }\end{array}$ & 44 & 26,99 & 6 & 3,68 & 8 & 4,91 & 68 & 41,72 & 37 & 22,70 \\
\hline 3 Ulceras en la boca & 50 & 30,67 & 13 & 7,98 & 10 & 6,13 & 84 & 51,53 & 6 & 3,68 \\
\hline 4 Tiene problemas de comida atorada entre dientes & 54 & 33,13 & 5 & 3,07 & 12 & 7,36 & 33 & 20,25 & 59 & 36,20 \\
\hline \multicolumn{11}{|l|}{ Limitación funcional } \\
\hline 5 Problemas para comer cosas duras & 35 & 21,47 & 11 & 6,75 & 11 & 6,75 & 83 & 50,92 & 23 & 14,11 \\
\hline $\begin{array}{l}6 \text { Tiene dolor de muelas al comer alimentos muy } \\
\text { fríos o muy calientes }\end{array}$ & 48 & 29,45 & 4 & 2,45 & 8 & 4,91 & 83 & 50,92 & 20 & 12,27 \\
\hline $\begin{array}{l}7 \text { Tiene problemas para pronunciar algunas } \\
\text { palabras }\end{array}$ & 16 & 9,82 & 10 & 6,13 & 4 & 2,45 & 126 & 77,30 & 7 & 4,29 \\
\hline $\begin{array}{l}8 \text { Le toma más tiempo al comer por problemas con } \\
\text { sus dientes }\end{array}$ & 21 & 12,88 & 11 & 6,75 & 3 & 1,84 & 114 & 69,94 & 14 & 8,59 \\
\hline \multicolumn{11}{|l|}{ Bienestar emocional } \\
\hline 9 Siente vergûenza de sus dientes & 29 & 17,79 & 6 & 3,68 & 12 & 7,36 & 98 & 60,12 & 18 & 11,04 \\
\hline 10 Se siente enojado por sus dientes & 16 & 9,82 & 8 & 4,91 & 5 & 3,07 & 129 & 79,14 & 5 & 3,07 \\
\hline $\begin{array}{l}11 \text { Le preocupa lo que sus compañeros piensen de } \\
\text { sus dientes }\end{array}$ & 27 & 16,56 & 7 & 4,29 & 7 & 4,29 & 100 & 61,35 & 22 & 13,50 \\
\hline 12 Sus dientes le causan desanimo o decepción & 25 & 15,34 & 5 & 3,07 & 8 & 4,91 & 114 & 69,94 & 11 & 6,75 \\
\hline \multicolumn{11}{|l|}{ Bienestar social } \\
\hline $\begin{array}{l}13 \text { Evita participar en clases por pena de pronuncia } \\
\text { mal algunas palabras }\end{array}$ & 11 & 6,75 & 7 & 4,29 & 4 & 2,45 & 136 & 83,44 & 5 & 3,07 \\
\hline 14 Sus dientes son motivo de burla & 22 & 13,50 & 7 & 4,29 & 8 & 4,91 & 120 & 73,62 & 6 & 3,68 \\
\hline 15 Sus dientes son motivo para evitar reír & 20 & 12,27 & 3 & 1,84 & 7 & 4,29 & 123 & 75,46 & 10 & 6,13 \\
\hline 16 Sus dientes son motivo de pelea & 11 & 6,75 & 4 & 2,45 & 2 & 1,23 & 140 & 85,89 & 6 & 3,68 \\
\hline
\end{tabular}

Tabla 1. Distribución de frecuencia, según ítems CPQ 8-10 corto en niños de la Escuela de Educación Básica "San Francisco de Peleusí de Azogues

\begin{tabular}{|c|c|c|c|c|c|c|c|c|c|c|c|}
\hline \multirow{3}{*}{$\begin{array}{l}\text { PREGUNTAS } \\
\text { Sintomatologia Oral }\end{array}$} & \multicolumn{11}{|c|}{ PCIONES DE RESPUESTA } \\
\hline & \multicolumn{2}{|c|}{ NUNCA } & \multicolumn{2}{|c|}{ ASI NUNCA } & \multicolumn{2}{|c|}{ A VECES } & \multicolumn{2}{|c|}{ ASI SIEMPRE } & \multicolumn{2}{|c|}{ SIEMPRE } & \multirow[b]{2}{*}{$\mathrm{p}^{*}$} \\
\hline & $\mathrm{n}$ & $\%$ & $n$ & $\%$ & $\mathrm{n}$ & $\%$ & $\mathrm{n}$ & $\%$ & $\mathrm{n}$ & $\%$ & \\
\hline 1. Dolor molar & 24 & 54,6 & 0 & 0 & 14 & 31,8 & 2 & 4,5 & 4 & 9,1 & 0,29 \\
\hline 2. Sangrado encias, al comer o cepillado dientes & 23 & 52,3 & 0 & 0 & 10 & 22,7 & 1 & 2,3 & 10 & 23 & 0,278 \\
\hline 3. Ulceras en la boca & 27 & 61,4 & 2 & 4,5 & 12 & 27,3 & 1 & 2,3 & 2 & 4,5 & 0,429 \\
\hline 4. Comida atorada entre dientes. & 7 & 15,9 & 2 & 4,5 & 10 & 22,7 & 1 & 2,3 & 24 & 55 & 0,03 \\
\hline \multicolumn{12}{|l|}{ Limitacion Funcional } \\
\hline 5. Problemas para comer cosas duras. & 18 & 40,9 & 5 & 11,4 & 8 & 18,2 & 4 & 9,1 & 9 & 21 & 0,231 \\
\hline 6. Dolor de muela al comer alimentos muy fríos o & 24 & 54,4 & 0 & 0 & 12 & 27,3 & 2 & 4,5 & 6 & 14 & 0,765 \\
\hline 7. Problemas para pronunciar algunas palabras & 34 & 77,3 & 4 & 9,1 & 3 & 6,8 & 0 & 0 & 3 & 6,8 & 0,436 \\
\hline 8. Tomar más tiempo comer, por probelmas en tus & 27 & 61,4 & 3 & 6,8 & 6 & 16,6 & 2 & 4,5 & 6 & 14 & 0,304 \\
\hline \multicolumn{12}{|l|}{ Bienestar Emocional } \\
\hline 9. Siente vergüenza & 25 & 56,8 & 4 & 9,1 & 5 & 11,4 & 3 & 6,8 & 7 & 16 & 0,107 \\
\hline 10. Se siente enojado por sus dientes. & 31 & 70,5 & 4 & 9,1 & 6 & 13,6 & 1 & 2,3 & 2 & 4,5 & 0,383 \\
\hline 11. Le preocupa lo que sus compañeros piensen & 28 & 63,6 & 2 & 4,5 & 5 & 11,4 & 0 & 0 & 9 & 21 & 0,215 \\
\hline 12. Dientes causan desánimo o decepción. & 32 & 72,7 & 1 & 2,3 & 5 & 11,4 & 2 & 4,5 & 4 & 9,1 & 0,858 \\
\hline \multicolumn{12}{|l|}{ Bienestar Social } \\
\hline $\begin{array}{l}\text { 13. Evitar participar en clase, por pena de } \\
\text { pronunciar mal algunas palabras. }\end{array}$ & 36 & 81,8 & 3 & 6,8 & 2 & 4,5 & 1 & 2,3 & 2 & 4,5 & 0,776 \\
\hline 14. Dientes son motivo de burla & 31 & 70,5 & 2 & 4,5 & 5 & 11,4 & 2 & 4,5 & 4 & 9,1 & 0,277 \\
\hline 15. Dientes son motivo de evitar reír & 32 & 72,7 & 0 & 0 & 4 & 9,1 & 3 & 6,8 & 5 & 11 & 0,263 \\
\hline 16. Dientes son motivo de pelea & 36 & 81,8 & 0 & 0 & 3 & 6,8 & 1 & 2,3 & 4 & 9,1 & 0,14 \\
\hline
\end{tabular}

Tabla 2. Identificación CVrSB de acuerdo al "CPQ corto"según edad en el rango de 6-7 años en la Escuela de Educación Básica "San Francisco de Peleusí de Azogues 


\begin{tabular}{|c|c|c|c|c|c|c|c|c|c|c|c|}
\hline \multicolumn{12}{|c|}{ OPCIONES DE RESPUESTA } \\
\hline PREGUNTAS & \multicolumn{2}{|c|}{ NUNCA } & \multicolumn{2}{|c|}{ CASI NUNCA } & \multicolumn{2}{|c|}{ A VECES } & \multicolumn{2}{|c|}{ CASI SIEMPRE } & \multicolumn{2}{|c|}{ SIEMPRE } & \multirow[b]{2}{*}{$\mathrm{p}^{*}$} \\
\hline Sintomatologia Oral & $\mathrm{n}$ & $\%$ & $n$ & $\%$ & $\mathrm{n}$ & $\%$ & $n$ & $\%$ & $\mathrm{n}$ & $\%$ & \\
\hline 1. Dolor molar & 56 & 47,1 & 6 & 5 & 31 & 26,1 & 15 & 12,6 & 11 & 9,2 & 0,29 \\
\hline $\begin{array}{l}\text { 2. Sangrado encias, al comer o } \\
\text { cepillado dientes }\end{array}$ & 45 & 37,8 & 6 & 5 & 34 & 28,6 & 7 & 5,9 & 27 & 22,7 & 0,278 \\
\hline 3. Ulceras en la boca & 57 & 47,9 & 11 & 9,2 & 38 & 31,9 & 9 & 7,8 & 4 & 3,4 & 0,429 \\
\hline 4. Comida atorada entre dientes. & 28 & 21,8 & 3 & 2,5 & 44 & 37 & 11 & 9,2 & 35 & 29,4 & 0,03 \\
\hline \multicolumn{12}{|l|}{ Limitacion Funcional } \\
\hline $\begin{array}{l}\text { 5. Problemas para comer cosas } \\
\text { duras. }\end{array}$ & 65 & 54,6 & 6 & 5 & 27 & 22,7 & 7 & 5,9 & 14 & 11,8 & 0,231 \\
\hline $\begin{array}{l}\text { 6. Dolor de muela al comer } \\
\text { alimentos muy fríos o muy }\end{array}$ & 59 & 99,6 & 4 & 3,4 & 36 & 30,3 & 6 & 5 & 14 & 11,8 & 0,765 \\
\hline $\begin{array}{l}\text { 7. Problemas para pronunciar } \\
\text { algunas palabras }\end{array}$ & 92 & 77,3 & 6 & 5 & 13 & 10,9 & 4 & 3,4 & 4 & 3,4 & 0,436 \\
\hline $\begin{array}{l}\text { 8. Tomar más tiempo comer, por } \\
\text { probelmas en tus dientes. }\end{array}$ & 87 & 73,1 & 8 & 6,7 & 15 & 12,6 & 1 & 0,8 & 8 & 6,7 & 0,304 \\
\hline \multicolumn{12}{|l|}{ Bienestar Emocional } \\
\hline 9. Siente vergüenza & 73 & 61,3 & 2 & 6,7 & 24 & 20,2 & 9 & 7,6 & 11 & 9,2 & 0,107 \\
\hline 10. Se siente enojado por sus & 98 & 82,4 & 4 & 3,4 & 10 & 8,4 & 4 & 3,4 & 3 & 2,5 & 0,383 \\
\hline $\begin{array}{l}\text { 11. Le preocupa lo que sus } \\
\text { compañeros piensen en sus }\end{array}$ & 72 & 60,5 & 5 & 4,2 & 22 & 18,5 & 7 & 5,9 & 13 & 10,9 & 0,215 \\
\hline $\begin{array}{l}\text { 12. Dientes causan desánimo o } \\
\text { decepción. }\end{array}$ & 82 & 68,9 & 4 & 4,4 & 20 & 16,8 & 6 & 5 & 7 & 5,9 & 0,858 \\
\hline \multicolumn{12}{|l|}{ Bienestar Social } \\
\hline $\begin{array}{l}\text { 13. Evitar participar en clase, por } \\
\text { pena de pronunciar mal algunas } \\
\text { palabras. }\end{array}$ & 100 & 98 & 4 & 3,4 & 9 & 7,8 & 3 & 2,5 & 3 & 2,5 & 0,776 \\
\hline 14. Dientes son motivo de burla & 89 & 74,8 & 5 & 4,2 & 17 & 14,3 & 6 & 5 & 2 & 2,7 & 0,277 \\
\hline 15. Dientes son motivo de evitar & 91 & 76,5 & 3 & 2,5 & 16 & 13,4 & 4 & 3,4 & 5 & 4,2 & 0,263 \\
\hline 16. Dientes son motivo de pelea & 104 & 87,4 & 4 & 3,4 & 8 & 6,7 & 1 & 0,8 & 2 & 1,7 & 0,14 \\
\hline
\end{tabular}

n: Frecuencia Absoluta \%: Frecuencia Relativa p:Significancia Estadistica $\quad$ *: Prueba de Chi-cuadrado

Tabla 3. Evaluación CVrSB de acuerdo al "CPQ corto"según edad en el rango de 8-10 años en la Escuela de Educación Básica "San Francisco de Peleusí de Azogues".

la dimensión más afectada fue la de Sintomatología Oral: en cuanto a la edad el grupo de 8 a 10 años aportó de mejor forma en la aplicación del instrumento indicando que la dimensión más afectada fue Sintomatología Oral lo que no ocurrió en el grupo de 6 a 7 años por las dificultades en la comprensión del cuestionario, en cuanto al sexo, el femenino presentó más problemas de salud oral afectando su calidad de vida.

\section{Referencias}

1 Aroila R. Calidad de vida: una definición integradora. Rev Latinoam Psicol. 2003;35(2):161-164.

2 Zacca González G, Rosales S, Mojáiber de la Peña A, others. Situación de salud bucal de la población cubana: Estudio comparativo según provincias, 1998. Rev Cuba Estomatol. 2001;38(2):90-110.

3 Moreno X, Vera C, Cartes R. Impacto de la Salud Bucal en la calidad de vida de los escolares de 11 a 14 años. Licantén, 2013. Revista Clínica de Periodoncia, Implantología y Rehabilitación Oral. 2014: 142-148.

4 Piovesan C, Batista A, Ferreira F, Ardenghi T. Oral healthrelated quality of life in children: Conceptual issues. Rev Odonto Cienc. 2009; 24(1): 81-85.

5 Santos Rita D, María I. Analisis de salud oral y su impacto en la calidad de vida de la población preescolar del municipio de Cascais, Portugal, en 2012. [tesis doctoral]. Cascais: Universidad de Sevilla, Departamento de Estomatología; 2015.
6 Sheiham A. Oral health, general health and quality of life. Bulletin of the World Health Organization. 2005; 83(9): 641-642.

7 Aldrigui J, Abanto J, Carvalho T, Mendes F, Wanderley M, Bönecker $M$ et al. Impact of traumatic dental injuries and malocclusions on quality of life of young children. Health Qual Life Outcomes. 2011; 9: 78.

8 Echeverría S, Henríquez E, Sepúlveda R, Barra P. Caries temprana de la infancia severa: impacto en la calidad de vida relacionada a la salud oral de los niños preescolares. Rev Dent Chile. 2010; 101(2):15-21.

9 Montero J. Calidad de vida oral en población general [tesis doctoral]. Universidad de Granada: Facultad de Odontología; 2006.

10 Al-Omiri M, Karasneh J. Relationship between oral healthrelated quality of life, satisfaction, and personality in patients with prosthetic rehabilitations. J Prosthodont. Jan. 2010;19(1):2-9.

11 Salinas P, Farías A, González X, Rodríguez C. Calidad de vida relacionada en salud: Concepto y evaluación en pacientes con ventilación mecánica no invasiva. Neumol Pediatr 2008; 3 (Supl): 34-9.

12 Wogelius P, Gjørup H, Haubek D, Lopez R, Poulsen S. Development of Danish version of child oral-health-related quality of life questionnaires (CPQ 8-10 \& CPQ 11-14 BMC Oral Health.2009;9:11.

13 Muñoz P, Aguilar F, de la Fuente Hernández J, Shimada H, Acosta L. Calidad de vida relacionada con la salud bucal en 


\begin{tabular}{|c|c|c|c|c|c|c|c|c|c|c|c|}
\hline \multicolumn{12}{|c|}{ OPCIONES DE RESPUESTA } \\
\hline \multirow{2}{*}{$\begin{array}{l}\text { PREGUNTAS } \\
\text { Sintomatologia Oral }\end{array}$} & \multicolumn{2}{|c|}{ NUNCA } & \multicolumn{2}{|c|}{ CASI NUNCA } & \multicolumn{2}{|c|}{ A VECES } & \multicolumn{2}{|c|}{ CASI SIEMPRE } & \multicolumn{2}{|c|}{ SIEMPRE } & \multirow[b]{2}{*}{$p^{*}$} \\
\hline & $\mathrm{n}$ & $\%$ & $\mathrm{n}$ & $\%$ & $\mathrm{n}$ & $\%$ & $\mathrm{n}$ & $\%$ & $\mathrm{n}$ & $\%$ & \\
\hline 1. Dolor molar & 39 & 54,2 & 3 & 4,2 & 19 & 26,4 & 6 & 8,3 & 5 & 6,9 & 0,719 \\
\hline $\begin{array}{l}\text { 2. Sangrado encias, al comer } \\
\text { o cepillado dientes }\end{array}$ & 32 & 44,4 & 5 & 6,9 & 17 & 23,6 & 2 & 2,8 & 16 & 22,2 & 0,222 \\
\hline 3. Ulceras en la boca & 33 & 45,8 & 5 & 6,9 & 24 & 33,3 & 4 & 5,6 & 6 & 0,3 & 0,067 \\
\hline \multicolumn{12}{|l|}{ 4. Comida atorada entre } \\
\hline \multicolumn{12}{|l|}{ Limitacion Funcional } \\
\hline $\begin{array}{l}\text { 5. Problemas para comer } \\
\text { cosas duras. }\end{array}$ & 43 & 59,7 & 3 & 4,2 & 13 & 18,1 & 2 & 2,8 & 11 & 15,3 & 0,132 \\
\hline $\begin{array}{l}\text { 6. Dolor de muela al comer } \\
\text { alimentos muy fríos o muy } \\
\text { calientes. }\end{array}$ & 37 & 51,4 & 2 & 2,8 & 20 & 27,8 & 5 & 6,9 & 8 & 11,1 & 0,842 \\
\hline $\begin{array}{l}\text { 7. Problemas para } \\
\text { pronunciar algunas palabras }\end{array}$ & 52 & 72,2 & 6 & 6,9 & 5 & 6,9 & 4 & 5,6 & 6 & 8,3 & 0,021 \\
\hline $\begin{array}{l}\text { 8. Tomar más tiempo comer, } \\
\text { por probelmas en tus } \\
\text { dientes. }\end{array}$ & 49 & 68,1 & 6 & 8,3 & 9 & 12,5 & 0 & 0 & 8 & 11,1 & 0,421 \\
\hline \multicolumn{12}{|l|}{ Bienestar Emocional } \\
\hline 9. Siente vergüenza & 45 & 62,5 & 2 & 2,8 & 15 & 20,8 & 4 & 5,6 & 6 & 8,3 & 0,643 \\
\hline $\begin{array}{l}\text { 10. Se siente enojado por } \\
\text { sus dientes. }\end{array}$ & 62 & 86,1 & 2 & 2,8 & 5 & 6,9 & 1 & 1,4 & 2 & 2,8 & 0,369 \\
\hline 11. Le preocupa lo que sus & & & & & & & & & & & \\
\hline $\begin{array}{l}\text { compañeros piensen en sus } \\
\text { dientes. }\end{array}$ & 47 & 65,3 & 2 & 2,8 & 14 & 19,4 & 4 & 5,6 & 5 & 6,9 & 0,182 \\
\hline $\begin{array}{l}\text { 12. Dientes causan } \\
\text { desánimo o decepción. }\end{array}$ & 52 & 72,2 & 2 & 2,8 & 12 & 16,7 & 3 & 4,2 & 3 & 4,2 & 0,791 \\
\hline \multicolumn{12}{|l|}{ Bienestar Social } \\
\hline $\begin{array}{l}\text { 13. Evitar participar en clase, } \\
\text { por pena de pronunciar mal } \\
\text { algunas palabras. }\end{array}$ & 63 & 87,5 & 2 & 2,8 & 4 & 5,6 & 1 & 1,4 & 2 & 2,8 & 0,763 \\
\hline $\begin{array}{l}\text { 14. Dientes son motivo de } \\
\text { burla }\end{array}$ & 52 & 72,2 & 1 & 1,4 & 15 & 20,8 & 2 & 2,8 & 2 & 2,8 & 0,057 \\
\hline $\begin{array}{l}\text { 15. Dientes son motivo de } \\
\text { evitar reír }\end{array}$ & 55 & 76,4 & 2 & 2,8 & 9 & 12,5 & 2 & 2,8 & 4 & 5,6 & 0,845 \\
\hline $\begin{array}{l}\text { 16. Dientes son motivo de } \\
\text { pelea }\end{array}$ & 56 & 77,8 & 2 & 2,8 & 9 & 12,5 & 0 & 0 & 5 & 6,9 & 0,013 \\
\hline
\end{tabular}

Tabla 4. Análisis del CVrSB de acuerdo al "CPQ corto"según el sexo masculino en la Escuela de Educación Básica "San Francisco de Peleusí de Azogues 


\begin{tabular}{|c|c|c|c|c|c|c|c|c|c|c|c|}
\hline \multirow{3}{*}{ PREGUNTAS } & \multicolumn{10}{|c|}{ OPCIONES DE RESPUESTA } & \multirow[b]{3}{*}{$\mathbf{p}^{*}$} \\
\hline & \multicolumn{2}{|c|}{ NUNCA } & \multicolumn{2}{|c|}{$\begin{array}{l}\text { CASI } \\
\text { NUNCA }\end{array}$} & \multicolumn{2}{|c|}{ A VECES } & \multicolumn{2}{|c|}{$\begin{array}{l}\text { CASI } \\
\text { SIEMPRE }\end{array}$} & \multicolumn{2}{|c|}{ SIEMPRE } & \\
\hline & $\mathbf{n}$ & $\%$ & $\mathbf{N}$ & $\%$ & $\mathbf{n}$ & $\%$ & $\mathbf{n}$ & $\%$ & $\mathbf{n}$ & $\%$ & \\
\hline \multicolumn{12}{|l|}{ Sintomatología Oral } \\
\hline 1. Dolor molar & 41 & 45,1 & 3 & 3,3 & 26 & 28,6 & 11 & 12,1 & 10 & 11 & 0,719 \\
\hline $\begin{array}{l}\text { 2. Sangrado encías, al comer o } \\
\text { cepillado dientes }\end{array}$ & 36 & 39,6 & 1 & 1,7 & 27 & 29,7 & 6 & 6.6 & 21 & 23,1 & 0,222 \\
\hline 3. Ulceras en la boca & 51 & 56 & 8 & 8,8 & 26 & 28,6 & 6 & 6,6 & 0 & 0 & 0,067 \\
\hline $\begin{array}{l}\text { 4. Comida atorada entre dientes } \\
\text { Limitación Funcional }\end{array}$ & 17 & 18,7 & 3 & 3,3 & 25 & 27,5 & 7 & 7.7 & 39 & 42,9 & 0,305 \\
\hline $\begin{array}{l}\text { 5. Problemas para comer cosas } \\
\text { duras }\end{array}$ & 40 & 44 & 8 & 8,8 & 24,2 & 9 & 9,9 & 12 & 13,2 & 11,8 & 0,132 \\
\hline $\begin{array}{l}\text { 6. Dolor de muela al comer } \\
\text { alimentos muy frios o muy } \\
\text { calientes }\end{array}$ & 46 & 50,5 & 2 & 2,2 & 28 & 30,8 & 3 & 3,3 & 12 & 13,2 & 0,842 \\
\hline $\begin{array}{l}\text { 7. Problemas para pronunciar } \\
\text { algunas palabras }\end{array}$ & 74 & 81,3 & 5 & 5,5 & 11 & 12,5 & 0 & 0 & 1 & 1,1 & 0,021 \\
\hline $\begin{array}{l}\text { 8. Toma más tiempo comer, por } \\
\text { problemas en tus dientes } \\
\text { Bienestar Emocional }\end{array}$ & 65 & 71,4 & 5 & 5,5 & 12 & 13,2 & 3 & 3.3 & 6 & 6.6 & 0,421 \\
\hline $\begin{array}{l}\text { 9. Siente vergüenza de sus } \\
\text { dientes }\end{array}$ & 53 & 58,2 & 4 & 4,1 & 14 & 15,4 & 8 & 8,8 & 12 & 13,2 & 0,643 \\
\hline $\begin{array}{l}\text { 10. Se siente enojado por sus } \\
\text { dientes }\end{array}$ & 67 & 73,6 & 6 & 6,6 & 11 & 12,1 & 4 & 4,4 & 3 & 3,3 & 0,369 \\
\hline $\begin{array}{l}\text { 11. Le preocupa lo que sus } \\
\text { compañeros piensen de sus } \\
\text { dientes }\end{array}$ & 53 & 58,2 & 5 & 5,5 & 13 & 14,3 & 3 & 3,3 & 17 & 18,6 & 0,182 \\
\hline $\begin{array}{l}\text { 12. Dientes causan desánimo o } \\
\text { decepción } \\
\text { Bienestar Social }\end{array}$ & 62 & 68,1 & 3 & 3,3 & 13 & 14,3 & 5 & 5,5 & 8 & 8,8 & 0,791 \\
\hline $\begin{array}{l}\text { 13. Evita participar en clase, por } \\
\text { pena de pronunciar mal algunas } \\
\text { palabras }\end{array}$ & 73 & 80,2 & 5 & 5,5 & 7 & 7,7 & 3 & 3,3 & 3 & 3,3 & 0,763 \\
\hline 14. Dientes son motivo de burla & 68 & 74,7 & 6 & 6,6 & 7 & 7,7 & 6 & 6,6 & 4 & 4,4 & 0,057 \\
\hline $\begin{array}{l}\text { 15. Dientes son motivo de evitar } \\
\text { reír }\end{array}$ & 68 & 74,7 & 1 & 1,1 & 11 & 12,1 & 5 & 5,5 & 6 & 6,6 & 0,845 \\
\hline 16. Dientes son motivo de pelea & 84 & 92,3 & 2 & 2,2 & 2 & 2,2 & 2 & 2,2 & 1 & 1,1 & 0,013 \\
\hline
\end{tabular}

Tabla 5. Identificación CVrSB de acuerdo al "CPQ corto"según el sexo femenino en la Escuela de Educación Básica "San Francisco de Peleusí de Azogues".

niños. Salud i Ciencia. 2014; 20(8):846-51.

14 Barbosa T, Tureli M, Gavião M. Validity and reliability of the Child Perceptions Questionnaires. BMC Oral Health 2009, 9: 13. 10.1186/1472-6831-9-13.

15 Zhang M, Mcgrath C, Hägg U. The impact of malocclusion and its treatment on quality of life: a literature review. Int J Paediatr Dent. 2006; 16: 381-387.

16 Aguilar F, Irigoyen M. Validation of the CPQ 8-10ESP in Mexican School children in urban areas. Med Oral Patol Oral Cir Bucal. 2011 May 1;16 (3): e430-5.

Recibido: 29 de enero de 2018

Aceptado: 20 de febrero de 2018 
